\title{
El papel de los márgenes extensivo e intensivo en el crecimiento de la I+D durante la crisis $(2007-2011)^{*}$
}

\author{
Dolores Añón Higón \\ Juan A. Máñez \\ Juan A. Sanchis-Llopis \\ Universitat de València y ERICES \\ Miguel C. Manjón \\ Universitat Rovira i Virgili y CREIP
}

\section{Resumen}

En este trabajo se analiza el crecimiento del gasto en $I+D$ de las empresas manufactureras españolas durante la reciente crisis, a través de su descomposición en tres componentes que recogen la entrada de empresas en la realización de actividades de $I+D$, su persistencia y su nivel de intensificación. Utilizando datos de la Encuesta sobre Estrategias Empresariales y centrándonos en el período 2007-2011, los resultados indican que la importancia relativa de cada componente difiere según el tamaño de las empresas y el sector tecnológico al que pertenecen. Las grandes perjudicadas por la crisis han sido las empresas pequeñas, que han visto, salvo las pertenecientes al sector de alta tecnología, reducir considerablemente sus inversiones en $I+D$. El principal determinante de este deterioro ha sido el margen intensivo.

Palabras clave: $I+D$, margen extensivo, margen intensivo, crisis.

Clasificación JEL: O3.

\begin{abstract}
In this study we analyse the growth of $R \& D$ spending of Spanish manufacturing firms during the recent crisis, through its decomposition into three components that gather the initiation in the performance of $R \& D$ activities, the persistence of such activities and their level of deepening. Using data from the Survey on Business Strategies for the period 1990-2006, the results indicate that the relative importance of each component differs according to the size of companies and the technological sector to which they belong. The most adversely affected by the crisis have been small companies, which have experienced, except for those belonging to the high-tech sector, a significant reduction on their $R \& D$ investments. The main determinant of this deterioration has been the intensive margin.
\end{abstract}

Keywords: $R \& D$, extensive margin, intensive margin, crisis.

JEL classification: 03 .

* Los autores agradecen los comentarios recibidos de un evaluador anónimo y la financiación recibida del Ministerio de Ciencia y Tecnología (proyecto ECO2014-55745-R y ECO2014-55553-P), BBVA 2014 y de la Generalitat Valenciana (proyecto PROMETEOII, 2014/054). También les gustaría agradecer a la Fundación SEPI por proporcionarnos los datos. Cualquier error que persista es responsabilidad de los autores. 


\section{Introducción}

Aumentar el gasto en investigación y desarrollo (I+D) es uno de los principales objetivos en la estrategia de largo plazo de la política europea. Así, en 2010, los miembros de la Unión Europea (UE) ratificaron el compromiso de aumentar el gasto en I+D de Europa al 3 por 100 del PIB como uno de los objetivos claves de la estrategia «Europa 2020». En promedio, y según datos del año 2012 de la OCDE (2014), en la UE-28 el gasto en I+D representa el 2 por 100 del PIB, por debajo del nivel de EE.UU. (2,8 por 100), Japón ( 3,3 por 100) y al mismo nivel que China (2 por 100). Los datos para España muestran una cifra significativamente inferior (13 por 100 del PIB). A pesar de los esfuerzos realizados desde mediados de la década de los noventa para acercarnos a la media europea, la crisis interrumpió este proceso de convergencia. Así, desde el inicio de la crisis los indicadores básicos de la actividad de investigación, desarrollo e innovación (I+D+i) en España no han dejado de empeorar (COTEC, 2014). En este estudio nos centraremos en el comportamiento del gasto privado en I+D en este período, analizando sus principales componentes en una muestra representativa del sector manufacturero español extraída de la Encuesta sobre Estrategias Empresariales (ESEE) para el período 2007-2011.

La disponibilidad de datos desagregados a nivel de empresa ha aumentado el interés por analizar de forma más detallada la evolución de la inversión privada en I+D. En particular, el objetivo de este estudio es analizar el crecimiento o deterioro de la inversión privada en I+D en el sector manufacturero español a través de su descomposición en los márgenes extensivo e intensivo, durante el período de recesión. Por una parte, el margen extensivo recoge la evolución de los gastos en I+D a través del número de empresas que inician tales actividades. Por otra parte, el margen intensivo puede descomponerse en dos términos distintos: persistencia en la realización de actividades de I+D de aquellas empresas que ya las han iniciado; e, intensificación del esfuerzo innovador (aumento o disminución del gasto en I+D) por parte de aquellas que ya realizan dichas actividades. Dada la composición de nuestra muestra, analizaremos los márgenes por sectores tecnológicos, utilizando como criterio de agrupación la intensidad tecnológica de la industria (industrias de intensidad tecnológica alta, media y baja).

La literatura que analiza la distinción entre el margen intensivo y extensivo se ha centrado principalmente en el estudio de los volúmenes de comercio (Besedes y Prusa, 2007) o en la adopción de nuevas tecnologías (Comín et al., 2008). Con la excepción de Añón et al. (2011), no existen otros estudios que analicen el crecimiento de la inversión privada en I+D utilizando la descomposición de dicho crecimiento en el margen intensivo y extensivo. Los trabajos existentes se han centrado básicamente en el análisis de los cambios a través del tiempo de la inversión en I+D y en el número de empresas innovadoras, considerando implícitamente la intensificación del esfuerzo innovador y obviando el papel de la persistencia en la realización de actividades de I+D. 
Paralelamente, buena parte de las políticas de promoción de la I+D buscan aumentar el número de empresas que comienzan a realizar actividades de I+D (es decir, se concentran en el margen extensivo). Sin embargo, estas políticas, en muchas ocasiones, tienen un alcance limitado ya que, en general, es la intensificación de estas actividades (es decir, el margen intensivo), lo que produce mejores resultados en términos de invenciones, patentes u otras medidas de resultados de la innovación. En el caso español, los resultados de Añón et al. (2011) y los que se obtienen en este trabajo, sugieren que no tener en cuenta el componente de persistencia puede tener consecuencias en el análisis y, por tanto, en el diseño de las políticas de incentivos.

Esta evidencia se basa en dos hipótesis de trabajo que se proponen en este estudio: (i) esperamos que los gastos en I+D de una empresa que comienza tales actividades sean relativamente bajos en los primeros años y aumenten conforme aumente el número de períodos que la empresa realice ininterrumpidamente dicha actividad; y, (ii) la probabilidad de que un episodio de I+D termine disminuye con la duración de dicho episodio (Máñez et al., 2015)ํ․

Estas dos hipótesis implican que la intensificación del esfuerzo innovador es más probable (o solo es posible) cuando hay persistencia. En otras palabras, la intensificación está condicionada a la persistencia. Es más, cuanto mayores sean las tasas de persistencia, mayor será el crecimiento de los gastos en I+D, incluso en ausencia de intensificación.

El resto de este artículo se organiza en las siguientes secciones. En la sección 2 se revisa la literatura más relevante. En la sección 3 se describen los datos así como la representatividad de la muestra. La sección 4 presenta evidencia descriptiva de ambos márgenes. En la sección 5 se describe la metodología utilizada para descomponer el crecimiento de la I+D, se analizan los resultados de su aplicación a los datos descritos en la sección 3 y se presentan algunos ejercicios de simulación. Finalmente, la sección 6 contiene las principales conclusiones del estudio y las implicaciones de política económica que se derivan de los resultados obtenidos.

\section{Revisión de la literatura}

La importancia relativa del margen extensivo frente al margen intensivo ha sido un tema de considerable debate en la literatura sobre el comercio internacional (Besedes y Prusa, 2007). Aunque hay estudios que hacen hincapié en la importancia del margen extensivo para explicar el crecimiento del volumen de comercio de un país (Hummels y Klenow, 2005), estudios recientes destacan el papel desempeñado por el margen intensivo (Bernard et al., 2009; Besedes y Prusa, 2007; Help-

\footnotetext{
${ }^{1}$ En este trabajo se defiine como episodio de $\mathrm{I}+\mathrm{D}$ el número de años que una empresa realiza ininterrumpidamente actividades de I+D.
} 
man et al., 2008) ${ }^{2}$. Estos trabajos concluyen que el crecimiento del comercio proviene principalmente de las relaciones comerciales existentes más que de nuevas relaciones comerciales (margen extensivo).

Resulta interesante subrayar, no obstante, que en estos estudios sobre las diferencias entre los márgenes extensivo e intensivo, los componentes de persistencia y de intensificación del margen intensivo no siempre se analizan de forma independiente. En particular, los estudios tienden a centrarse en el componente de intensificación sin considerar el componente de persistencia. Esta omisión, sin embargo, no es neutral. Por el contrario, tal y como apuntan Besedes y Prusa (2007), obviar la persistencia tiene importantes consecuencias sobre el análisis del crecimiento de la variable de interés, puesto que la persistencia es una condición necesaria para que se pueda producir la intensificación y, además, mayor persistencia per se resultará en un mayor crecimiento incluso aunque no se produzca la intensificación.

En relación con los estudios sobre el crecimiento de la I+D, es importante señalar que, salvo el estudio de Añón et al. (2011), no existe otro estudio que integre los distintos aspectos de los márgenes intensivo y extensivo para explicar el crecimiento de la I+D privada. Los trabajos existentes analizan cambios en el crecimiento de la I+D conducidos por el número de empresas que llevan a cabo estas actividades considerando implícitamente la intensificación en dichas actividades y sin tener en cuenta el componente de la persistencia. A pesar de que desde un punto de vista teórico se reconoce que la innovación es inherentemente un proceso dinámico entre empresas heterogéneas (Blundell et al., 1995), la evidencia empírica a nivel de empresa sobre la persistencia en la realización de actividades de I+D es escasa. ${ }^{3}$ En particular, la persistencia en la innovación ha sido analizada desde el punto de vista de los resultados del proceso de innovación ${ }^{4}$. Sin embargo, pocos estudios han analizado la persistencia desde el punto de vista de los inputs, es decir, persistencia en las actividades de I+D (son excepciones los trabajos de Máñez et al., 2009, 2015; Peters, 2009 y; Cuervo-Cazurra y Un, 2010). Estos estudios encuentran que las actividades de I+D muestran una elevada persistencia a nivel de empresa y que dicha persistencia difiere entre sectores tecnológicos. Nuestra contribución a esta literatura es analizar el crecimiento o deterioro de la inversión en I+D en las

\footnotetext{
${ }^{2}$ La distinción entre estos márgenes también ha sido analizada en la literatura que estudia la adopción y difusión de nuevas tecnologías (COMíN et al., 2008), aunque con un enfoque distinto. En esta literatura, el margen extensivo se refiere a la difusión de nuevas tecnologías, mientras que el margen intensivo se relaciona al grado en que se utiliza una determinada tecnología. De nuevo, estos estudios resaltan la importancia del margen intensivo a la hora de explicar el crecimiento en la utilización o adopción de la tecnología.

${ }_{3}^{3}$ Existen varias razones teóricas que explican la persistencia en las actividades de innovación: los efectos del aprendizaje (ROSENBERG, 1976); las teorías de que el éxito da lugar a más éxito (MANSFIELD, 1968) o la existencia de costes hundidos (SUTTON, 1991). Véase MÁÑEZ et al. (2009, 2015) para una explicación con detalle de dichas razones.

${ }^{4}$ Sin embargo, no existen resultados concluyentes. Así, mientras que los estudios que utilizan patentes encuentran bajos niveles de persistencia (CEFIS, 2003; GEROSKI et al., 1997), aquellos que utilizan modelos de count para analizar el número de innovaciones obtienen resultados mixtos: por una parte DUGUET y MONJON (2004) y ROGERS (2004) encuentran una elevada persistencia, por otra parte GEROSKI et al (1997) y RAYMOND et al. (2010) no encuentran evidencia de persistencia en la innovación para las empresas manufactureras del Reino Unido y de Holanda, respectivamente.
} 
manufacturas españolas a través de su descomposición en los márgenes extensivo e intensivo durante el período de recesión económica. Dicha descomposición se realiza por sectores tecnológicos (industrias de intensidad tecnológica alta, media y baja), distinguiendo en el margen intensivo la persistencia en la realización de actividades de I+D de aquellas empresas que ya las han iniciado y la intensificación del esfuerzo innovador (o desinversión) que supone el aumento (o disminución) del gasto en I+D por parte de aquellas que ya realizan dichas actividades.

\section{Los datos}

Los datos utilizados en este trabajo proceden de la Encuesta Sobre Estrategias Empresariales (ESEE), para el período 1990-2011. Este trabajo concentra en el período de la reciente crisis económica, 2007-2011 . La ESEE es una encuesta anual representativa de las empresas manufactureras españolas clasificadas por sectores industriales y por categorías de tamaño, que proporciona información exhaustiva a nivel de empresa, incluidos los gastos de I+D 6 . La ESEE es un panel incompleto, dado que algunas empresas dejan de operar en el mercado, cambian su actividad para realizar actividades no industriales, o deciden salirse de la encuesta. Sin embargo, se han realizado importantes esfuerzos para mantener la representatividad de la muestra a lo largo del tiempo.

\subsection{Representatividad}

En el Cuadro 1 se resumen los principales descriptivos (porcentaje de empleo, ventas y gastos de I+D), por categorías de tamaño. Estos descriptivos se presentan tanto para la muestra completa del año 2007, como para las empresas que permanecen en la muestra todos los años de nuestro análisis (muestra continua), distinguiendo entre empresas que realizan $\mathrm{I}+\mathrm{D}$ y empresas que no realizan dichas actividades (empresas sin $\mathrm{I}+\mathrm{D})$. Así, si nos centramos en la muestra de empresas que realizan $\mathrm{I}+\mathrm{D}$, obtenemos que, tanto para las empresas pequeñas (con menos de 200 trabajadores) como para las grandes, el porcentaje de empresas, tamaño, gastos de I+D sobre ventas, ventas y empleo son muy similares en las muestras completa y continua. También es importante resaltar que la mayoría de las empresas son empresas pequeñas (y medianas) y que la mayoría de las empresas grandes hacen $\mathrm{I}+\mathrm{D}$ (y esto no ocurre en el caso de las pequeñas).

\footnotetext{
5 Sin embargo, para analizar la duración de los episodios de I+D tenemos en cuenta todo el período temporal de la base de datos, es decir desde 1990 a 2011.

${ }^{6}$ Para más detalles sobre la encuesta, véase, por ejemplo, FARIÑAS y JAUMANDREU (1999). También se puede consultar en la página web http://www.funep.es/esee/sp/sinfo_que_es.asp..
} 
CUADRO 1

\section{REPRESENTATIVIDAD DE LAS MUESTRAS: EMPRESAS QUE REALIZAN ACTIVIDADES DE I+D VERSUS EMPRESAS QUE NO REALIZAN ACTIVIDADES DE I+D}

\begin{tabular}{|l|c|c|c|c|}
\hline \multirow{2}{*}{ Medias } & \multicolumn{2}{|c|}{$\begin{array}{c}\text { Muestra completa para } \\
\text { 2007 }\end{array}$} & \multicolumn{2}{c|}{$\begin{array}{c}\text { Muestra continua } \\
\text { 2007-2011 }\end{array}$} \\
\cline { 2 - 5 } & $\begin{array}{c}\text { Empresas } \\
\text { sin I+D }\end{array}$ & $\begin{array}{c}\text { Empresas } \\
\text { I+D }\end{array}$ & $\begin{array}{c}\text { Empresas } \\
\text { sin I+D }\end{array}$ & $\begin{array}{c}\text { Empresas } \\
\text { I+D }\end{array}$ \\
\hline 1. Empresas pequeñas & \multicolumn{4}{|c|}{} \\
\hline Número de empresas & 1.178 & 335 & 606 & 186 \\
\hline Porcentaje del total de la muestra & 77,86 & 22,14 & 76,52 & 23,48 \\
\hline Tamaño medio (núm. empleados) & 45,05 & 96,44 & 45,49 & 91,90 \\
\hline Gasto I+D /Ventas (media) (\%) & 0 & 2,17 & 0 & 1,95 \\
\hline Porcentaje del total de las ventas & 55,99 & 44,01 & 54,86 & 45,14 \\
\hline Porcentaje del total del empleo & 62,16 & 37,84 & 61,72 & 38,28 \\
\hline 2. Empresas grandes & \multicolumn{4}{|c|}{} \\
\hline Número de empresas & 144 & 352 & 65 & 171 \\
\hline Porcentaje del total de la muestra & 29,03 & 70,97 & 27,54 & 72,46 \\
\hline Tamaño medio (núm. empleados) & 504,45 & 891,31 & 473,57 & 995,36 \\
\hline Gasto I+D/Ventas (media) (\%) & 0 & 1,77 & 0 & 1,91 \\
\hline Porcentaje del total de las ventas & 15,56 & 84,43 & 13,75 & 86,24 \\
\hline Porcentaje del total del empleo & 18,8 & 81,2 & 15,31 & 84,69 \\
\hline
\end{tabular}

NOTAS: 1) Las empresas que realizan actividades de I+D son aquellas que reportan gastos positivos en I+D (frente a las empresas que no realizan actividades de I+D, que reportan gastos nulos). 2) La muestra completa en 2007 recoge a todas las empresas que responden en 2007. 3) La muestra de empresas que permanecen desde 2007-2011 compone la muestra continua (desde 2007). 4) El gasto $\mathrm{I}+\mathrm{D} /$ Ventas se calcula como el promedio de este ratio para todas las empresas.

Por su parte, en el Cuadro 2 se presentan las proporciones de empresas (pequeñas y grandes) para las industrias de tecnología baja, media y alta, para la muestra total en 2007 y para la muestra continua ${ }^{7}$. Para las empresas pequeñas se obtienen proporciones muy similares, mientras que para las grandes solo el porcentaje de empresas en

${ }^{7}$ La clasificación de las industrias en los 3 sectores de tecnología sigue el criterio de la OCDE (2003). Así, el sector de tecnología baja está formado por las industrias de bebidas, textiles y prendas de vestir, cuero y calzado, madera, industria del papel, sector de impresión y productos de impresión, productos minerales no-metálicos, productos metálicos, muebles y otros productos manufactureros. El sector de tecnología media se compone de las industrias de alimentos y tabaco, caucho y plásticos, metalurgia, maquinaria y equipo mecánico y motores y vehículos. Finalmente, el sector de tecnología alta está formado por las industrias de productos químicos, máquinas de oficina, electrónica, maquinaria y material eléctrico y otros equipos de transporte. 


\section{CUADRO 2}

\section{REPRESENTATIVIDAD DE LAS MUESTRAS POR TAMAÑO Y SECTOR TECNOLÓGICO (2007)}

\begin{tabular}{|c|c|c|c|c|c|}
\hline \multicolumn{2}{|c|}{ Tecnología baja } & \multicolumn{2}{c|}{ Tecnología media } & \multicolumn{2}{c|}{ Tecnología alta } \\
\hline $\begin{array}{c}\text { Muestra } \\
\text { completa } \\
\text { para 2007 }\end{array}$ & $\begin{array}{c}\text { Muestra } \\
\text { continua } \\
\mathbf{2 0 0 7 - 2 0 1 1}\end{array}$ & $\begin{array}{c}\text { Muestra } \\
\text { completa } \\
\text { para 2007 }\end{array}$ & $\begin{array}{c}\text { Muestra } \\
\text { continua } \\
\mathbf{2 0 0 7 - 2 0 1 1}\end{array}$ & $\begin{array}{c}\text { Muestra } \\
\text { completa } \\
\text { para 2007 }\end{array}$ & $\begin{array}{c}\text { Muestra } \\
\text { continua } \\
\mathbf{2 0 0 7 - 2 0 1 1}\end{array}$ \\
\hline \multicolumn{6}{|c|}{ Empresas pequeñas } \\
\hline 946 & 479 & 386 & 223 & 181 & 90 \\
\hline $65,52 \%$ & $60,48 \%$ & $25,51 \%$ & $25,51 \%$ & $11,96 \%$ & $11,36 \%$ \\
\hline \multicolumn{7}{|c|}{ Empresas grandes } \\
\hline 183 & 76 & 200 & 106 & 113 & 54 \\
\hline $36,90 \%$ & $32,20 \%$ & $40,32 \%$ & $44,92 \%$ & $22,78 \%$ & $22,88 \%$ \\
\hline
\end{tabular}

la industria de tecnología alta es similar en ambas muestras, ya que las empresas que operan en las industrias de tecnología baja se encuentran infrarrepresentadas en la muestra continua y las empresas que operan en sectores de tecnología media están sobrerrepresentadas.

Estos resultados nos llevan a considerar que la muestra que utilizamos para llevar a cabo nuestro análisis (muestra continua) es comparable con la muestra completa para las empresas pequeñas. Si bien existen algunas diferencias para las empresas grandes, obtenemos cifras comparables para las variables de interés en este estudio (en particular, el tamaño y los gastos en I+D sobre ventas) ${ }^{8}$.

\section{Márgenes extensivo e intensivo}

\subsection{Margen extensivo}

El margen extensivo en cada sector tecnológico se define como el número de empresas que comienzan a realizar actividades de I+D en un determinado año. Por lo tanto, una industria experimentará un cambio en el margen extensivo cuando una empresa que no llevaba a cabo actividades de I+D anteriormente comienza a realizar tales actividades en un determinado momento en el tiempo.

${ }^{8}$ Utilizando la muestra de empresas que colaboran de forma continua todos los años evitamos el problema que provocarían aquellas empresas que dejan de colaborar en la encuesta. Esto es así porque el interés de nuestro trabajo es conocer las empresas que empiezan o terminan sus actividades de I+D. En ocasiones, una empresa termina de hacer estas actividades porque la empresa cesa su actividad y, por tanto, sus actividades innovadoras. Consideramos que, dados los resultados de las pruebas de representatividad, seleccionar a las empresas que colaboran todo el período no afecta a nuestros resultados. 
El Cuadro 3 muestra una serie de estadísticos descriptivos que analizan la evolución del margen extensivo en el período 2007-2011, por grupos de tamaño (empresas grandes y pequeñas) y por sector de intensidad tecnológica (alta, media y baja). La primera columna del cuadro muestra la tasa de crecimiento del gasto privado en I+D (en términos reales), mientras que la segunda columna presenta la tasa de crecimiento del número de empresas innovadoras. Ambos estadísticos nos confirman un retroceso importante para las empresas pequeñas, tanto de la inversión en I+D como del número de empresas innovadoras, durante el período de recesión. Comparando estas dos columnas se observa además que la tasa de crecimiento del gasto en I+D en las empresas pequeñas cae menos que la tasa de crecimiento del número de empresas que realizan I+D (más relacionada con el margen extensivo). Por su parte, en relación con las empresas grandes se observa que la tasa de crecimiento del gasto en $\mathrm{I}+\mathrm{D}$, en este caso positiva aunque modesta, es mayor que la tasa de crecimiento del número de empresas que realizan $\mathrm{I}+\mathrm{D}$.

El desglose por sectores tecnológicos permite observar que en las empresas pequeñas los sectores de tecnología baja y media tienen tasas negativas en el crecimiento del gasto en I+D mayores a las tasas negativas del crecimiento del número de empresas. Sin embargo, el comportamiento para las empresas del sector de tecnología alta es distinto, ya que mientras la tasa de crecimiento del gasto en $\mathrm{I}+\mathrm{D}$ es positiva, el número de empresas que hacen I+D sufre una importante caída. Por su parte, en las empresas grandes se observa que el sector de tecnología baja tiene una tasa de crecimiento en el gasto en $\mathrm{I}+\mathrm{D}$ negativa mientras que la tasa de crecimiento del número de empresas que hacen I+D es positivo. En el sector de tecnología media las dos tasas de crecimiento son negativas, aunque la caída en el gasto en $\mathrm{I}+\mathrm{D}$ es mayor que la que se observa en el número de empresas. Finalmente, y de manera análoga a las pequeñas empresas, en el sector de tecnología alta se observa una tasa positiva en el crecimiento en el gasto en I+D y una tasa negativa en el número de empresas que realizan $\mathrm{I}+\mathrm{D}$.

Por lo que respecta a la comparación por grupos de tamaño, mientras que el grupo de empresas pequeñas muestra una caída importante en términos del número de empresas innovadoras para los tres sectores tecnológicos, el número de empresas grandes realizando actividades de I+D apenas cae o incluso aumenta. Por otra parte, para ambos grupos de empresas el crecimiento medio del gasto en $\mathrm{I}+\mathrm{D}$ es negativo en los sectores de tecnología media y baja y es positivo en el de tecnología alta, aunque es mayor en este último caso para las empresas grandes.

Las columnas 3 y 4 del Cuadro 3 proporcionan, para cada uno de los grupos de tamaño y sectores tecnológicos, el porcentaje de empresas que realizan actividades de I+D sobre el total de empresas existentes (potencial realizado) para los años 2007 y 2011, respectivamente. De estas cifras cabe destacar, en primer lugar, que la comparativa por grupos de tamaño muestra que el potencial realizado se ha reducido para las empresas pequeñas (de 23,48 por 100 a 20,58 por 100 ), mientras que ha crecido ligeramente para el grupo de empresas grandes (de 72,46 por 100 a 73,31 por 100). En segundo lugar, que la comparativa entre sectores tecnológicos muestra 


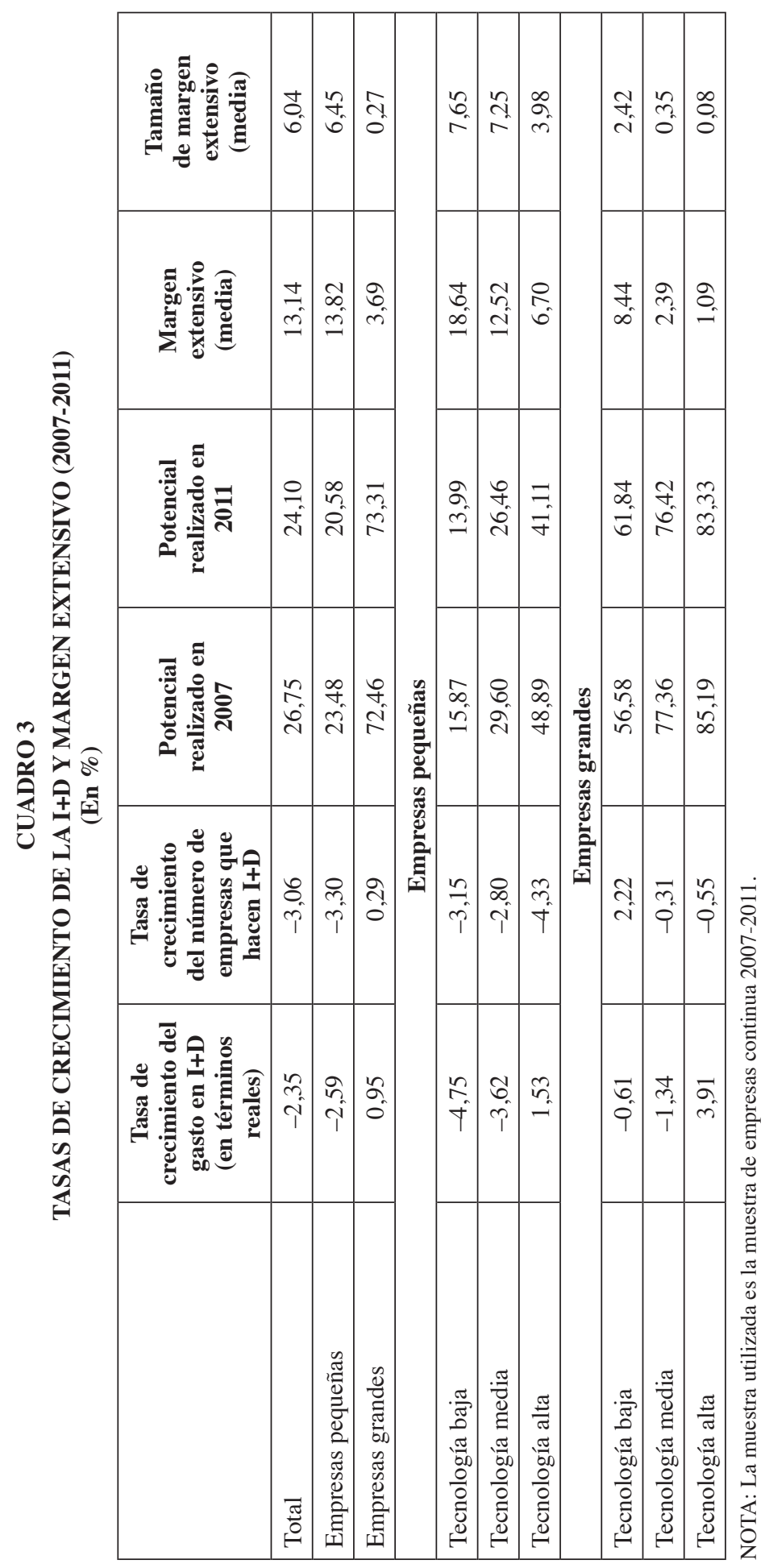


que para ambos grupos de empresas existe una correlación entre el porcentaje de empresas que realizan actividades de I+D y la intensidad tecnológica, tanto en 2007 como en 2011. En último término, las cifras ponen de manifiesto la existencia de un largo camino por recorrer en la inversión privada en I+D, especialmente en el grupo de empresas pequeñas (en 2011 el porcentaje de empresas pequeñas que realizaban I+D es del 20,58 por 100 y en el caso de las empresas grandes es del 73,31 por 100).

La quinta columna del Cuadro 3 informa sobre el margen extensivo a partir de la proporción de empresas que inician actividades de $\mathrm{I}+\mathrm{D}$ (número de empresas que inician actividades de $\mathrm{I}+\mathrm{D}$ en un determinado año sobre el total de empresas que realizan actividades de $\mathrm{I}+\mathrm{D}$ ese año, en media para el período considerado). Así, podemos observar que el margen extensivo es mayor para el grupo de empresas pequeñas que para el de grandes (13,82 por 100 y 3,69 por 100, respectivamente). Además, la diferencia entre empresas grandes y pequeñas es especialmente relevante para los sectores de intensidad tecnológica baja y media, para los que el margen extensivo en el caso de las pequeñas es del 18,64 por 100 y 12,52 por 100 , respectivamente (la diferencia para el caso del sector de tecnología alta, si bien apreciable, es menor). Estas diferencias sugieren que la evolución del margen extensivo es el factor más relevante en la diferente evolución del gasto en I+D de las empresas pequeñas frente a las grandes.

Por último, la columna 6 del Cuadro 3 ofrece una medida alternativa del tamaño del margen extensivo a partir del porcentaje medio del gasto en $\mathrm{I}+\mathrm{D}$ de las empresas que comienzan a realizar I+D sobre el total de gasto en I+D. Comparando las columnas 5 y 6 se observa que el peso relativo de las empresas que inician actividades de I+D sobre el total de empresas innovadoras es mayor que su aportación a los gastos en $\mathrm{I}+\mathrm{D}$, de lo que se deduce que el gasto medio en $\mathrm{I}+\mathrm{D}$ de las empresas que empiezan a realizar actividades de $\mathrm{I}+\mathrm{D}$ es considerablemente menor que el correspondiente al de las empresas que realizan dichas actividades de forma continua. Esta evidencia refuerza la idea de que la incorporación de nuevas empresas únicamente tendrá un impacto significativo sobre el crecimiento del gasto en I+D total de un país si estas empresas continúan realizando actividades de $\mathrm{I}+\mathrm{D}$ y aumentan su gasto con el paso del tiempo.

\subsection{Margen intensivo}

Para estudiar la evolución del margen intensivo analizamos tanto la duración de las empresas en la realización de actividades de I+D como la intensificación (aumento del gasto) en la realización de estas actividades por parte de aquellas empresas que ya las realizaban. 


\section{Persistencia}

Para un determinado año, podemos encontrar empresas con distinta «antigüedad» (años de realización ininterrumpida de actividades de $\mathrm{I}+\mathrm{D}$ ) en la realización de actividades de $\mathrm{I}+\mathrm{D}$, ya que algunas empresas realizan actividades de $\mathrm{I}+\mathrm{D}$ de forma continua, mientras que otras las realizan únicamente de forma esporádica. Es por ello que un proceso clave para nuestro análisis de la persistencia en la realización de actividades de I+D es la conversión de los datos anuales en períodos ininterrumpidos en la realización de actividades de I+D (episodio de I+D). Así, definimos un episodio de I+D como el número de años que una empresa realiza continuamente actividades de $\mathrm{I}+\mathrm{D}$ (es decir, tiene gastos positivos en la realización de actividades de I+D). En particular, para definir los episodios de I+D, tendremos en cuenta la información de las empresas desde $1990^{9}$. Una vez generada esta información, su tratamiento estadístico se realiza a partir de la estimación de funciones de supervivencia empíricas (de Kaplan-Meier), las cuales se presentan por grupos de tamaño (Gráfico 1) y sectores tecnológicos (Gráficos 2 y 3 ).

En el Gráfico 1 podemos observar que existen importantes diferencias entre las funciones de supervivencia de las empresas grandes y pequeñas. En primer lugar, observamos que la duración de los episodios de realización ininterrumpida de $\mathrm{I}+\mathrm{D}$ es significativamente mayor para las empresas grandes que para las pequeñas. La duración mediana de estos episodios es de 2 años para las empresas pequeñas en general (como se desprende del Gráfico 1$)^{10}$, mientras que las empresas grandes tienen episodios más largos, si bien su duración mediana varía en función de la intensidad tecnológica del grupo considerado (véase Gráfico 3) ${ }^{11}$.

\footnotetext{
9 En este estudio no es posible solucionar el problema de censura por la izquierda para los períodos de relación ininterrumpida de actividades de I+D.

${ }^{10}$ Esta duración mediana corresponde concretamente a aquellas que pertenecen a sectores de tecnología baja (véase Gráfico 2).

${ }^{11}$ La hipótesis nula de igualdad de las funciones de supervivencia por sectores tecnológicos se rechaza tanto para el grupo de empresas pequeñas $\left(\chi^{2}=17,16\right.$ y un $p$-value $\left.=0,000\right)$, como para las grandes $\left(\chi^{2}=13,79\right.$ y un $p$-value $\left.=0,001\right)$.
} 
GRÁFICO 1

FUNCIONES DE SUPERVIVENCIA DE LOS EPISODIOS DE I+D POR TAMAÑO (1990-2011)

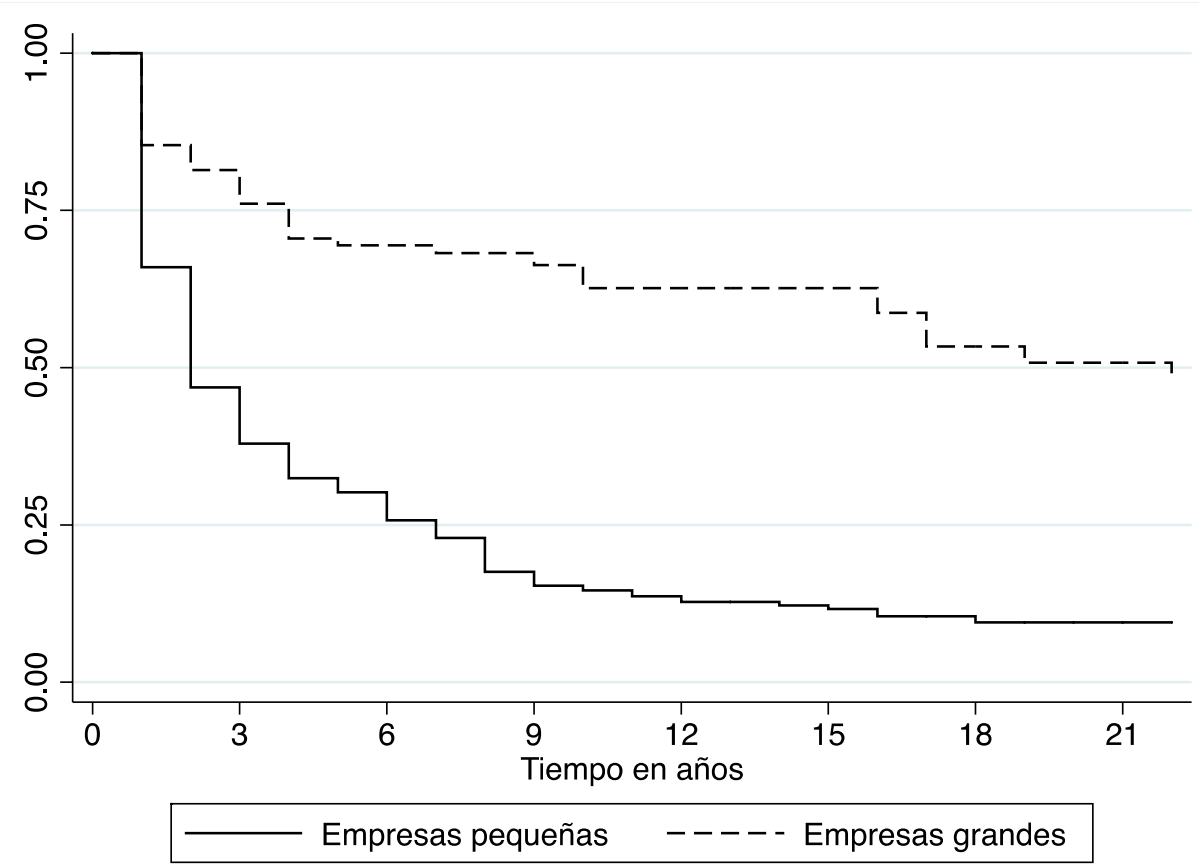

En segundo lugar, observamos que mientras que para las empresas grandes transcurridos 20 años el 50 por 100 de los episodios de I+D todavía continúan activos, para las empresas pequeñas este porcentaje se sitúa en un 10 por 100. De nuevo, estos porcentajes difieren según la intensidad tecnológica del sector, siendo mucho mayor el porcentaje de episodios que siguen activos cuando la intensidad tecnológica del sector es alta (véase Gráficos 2 y 3). 


\section{GRÁFICO 2}

\section{FUNCIONES DE SUPERVIVENCIA DE LOS EPISODIOS DE I+D PARA} EMPRESAS PEQUEÑAS POR SECTOR TECNOLÓGICO (1990-2011)

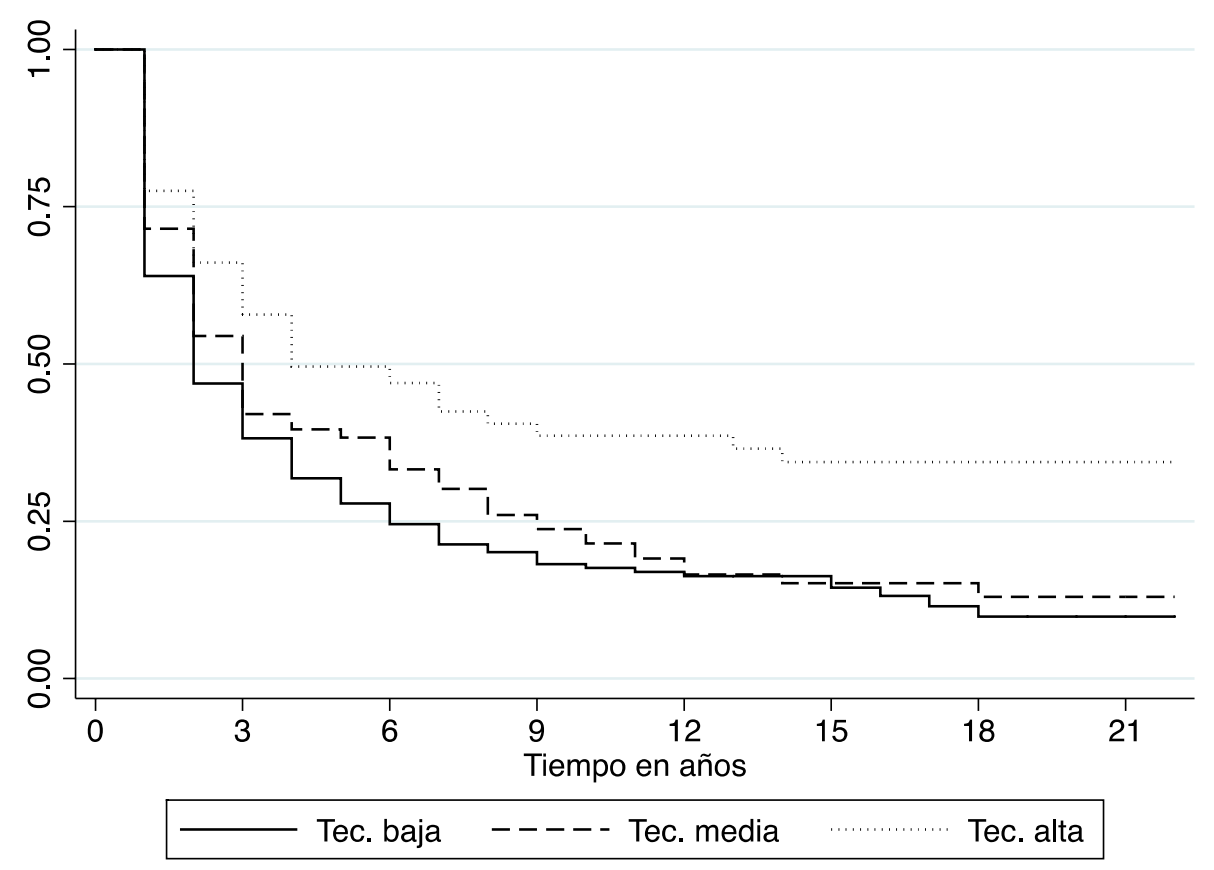

La baja tasa de supervivencia en la realización de actividades de I+D observada para las empresas pequeñas indica que hay que interpretar con cautela la contribución del margen extensivo al crecimiento del gasto en I+D de estas empresas. Nuestro análisis de duración pone de manifiesto que las ganancias en el margen extensivo de las empresas pequeñas se agotan transcurridos pocos años. En todo caso, las contribuciones del margen extensivo de las empresas grandes al crecimiento de la I+D son más duraderas. 


\section{GRÁFICO 3 \\ FUNCIONES DE SUPERVIVENCIA DE LOS EPISODIOS DE I+D PARA EMPRESAS GRANDES POR SECTOR TECNOLÓGICO (1990-2011)}

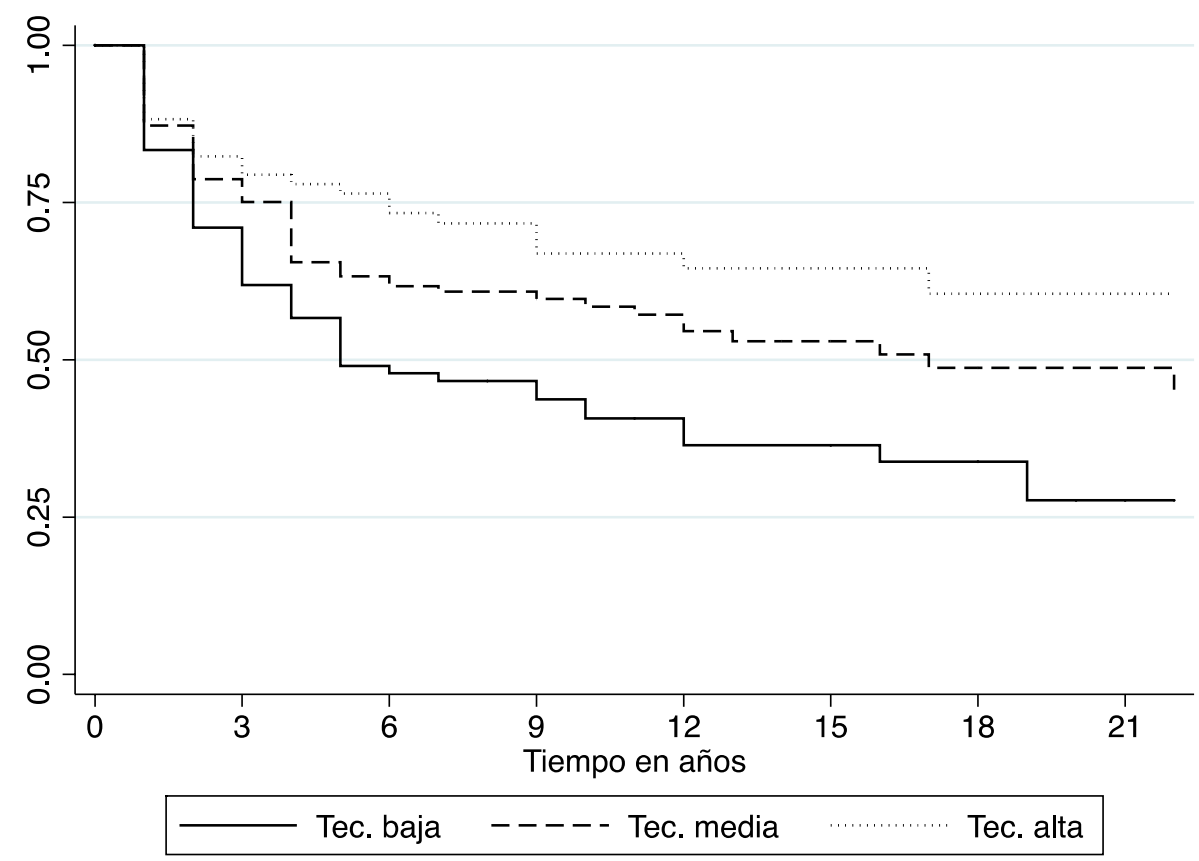

\section{Intensificación}

El análisis de la persistencia en la realización de actividades de I+D revela que dada la menor duración de los episodios de I+D entre las empresas pequeñas, muchas de ellas tendrán escasas oportunidades para intensificar su gasto en I+D (entendiendo por intensificar aumentar el gasto en I+D conforme aumenta la duración del episodio de I+D). Teniendo en cuenta este resultado, para el análisis del componente de intensificación nos centraremos en los episodios de I+D que empiezan, los que duran 4 o más años, y los que duran 10 o mas años, en 2007.

Las columnas 1 y 2 del Cuadro 4 muestran el porcentaje (número de episodios de I+D sobre los episodios activos) y el valor relativo de los episodios de I+D (valor de la I+D de los episodios sobre el valor de los episodios activos) que comienzan en 2007, respectivamente. Así, mientras que para las empresas grandes el porcentaje de episodios que comienzan en 2007 es del 8,77 por 100 (siendo los gastos en I+D de estos episodios el 0,64 por 100 del valor de los episodios activos) $)^{12}$, el de las empresas pequeñas es del 19,35 por 100 (siendo el valor relativo de estos episodios del 18,06 por 100).

${ }^{12}$ La mayor parte de las empresas grandes ya realizan I+D y gastan cantidades importantes en $\mathrm{I}+\mathrm{D}$, de ahí que las que empiezan son pocas en relación con las existentes y el valor de sus gastos en I+D sea también bajo. Lo contrario ocurre con las pequeñas, como se muestra a continuación. 


\section{CUADRO 4 \\ INTENSIFICACIÓN DEL GASTO EN I+D EN 2007 \\ (En \%)}

\begin{tabular}{|l|c|c|c|c|c|c|}
\hline \multirow{2}{*}{} & \multicolumn{2}{|c|}{ Año de inicio } & \multicolumn{2}{c|}{$\begin{array}{c}\text { Episodios de 4 } \\
\text { o más años }\end{array}$} & \multicolumn{2}{c|}{$\begin{array}{c}\text { Episodios de 10 } \\
\text { o más años }\end{array}$} \\
\cline { 2 - 7 } & Número & Valor $^{(1)}$ & Número & Valor $^{(1)}$ & Número & Valor $^{(1)}$ \\
\hline Todas \\
\hline Pequeñas & 19,35 & 18,06 & 38,71 & 46,36 & 25,81 & 32,12 \\
\hline Grandes & 8,77 & 0,64 & 60,23 & 59,88 & 38,01 & 52,55 \\
\hline \multicolumn{7}{|c|}{ Empresas pequeñas } \\
\hline Tecnología baja & 21,05 & 27,20 & 36,84 & 37,63 & 22,37 & 17,10 \\
\hline Tecnología media & 16,67 & 11,10 & 36,36 & 45,31 & 21,21 & 33,55 \\
\hline Tecnología alta & 20,45 & 13,07 & 45,45 & 61,06 & 38,64 & 53,23 \\
\hline \multicolumn{7}{|c|}{ Empresas grandes } \\
\hline Tecnología baja & 11,63 & 7,97 & 69,77 & 66,92 & 34,88 & 50,09 \\
\hline Tecnología media & 9,76 & 0,50 & 56,10 & 42,18 & 36,59 & 36,31 \\
\hline Tecnología alta & 4,35 & 0,33 & 58,70 & 84,07 & 43,48 & 75,39 \\
\hline
\end{tabular}

NOTA: ${ }^{(1)}$ Tasa media anual acumulativa.

En las columnas 3 y 4 se presentan el porcentaje y el valor relativo de los episodios de I+D que han durado al menos 4 años. Para las empresas grandes se observa que el porcentaje de los episodios de I+D que han durado al menos 4 años es bastante elevado $(60,23$ por 100$)$. Además, este porcentaje es prácticamente igual al valor relativo de estos episodios de I+D (59,88 por 100), lo que indica que conforme aumenta la duración de estos episodios, el componente de intensificación empieza a ser relevante. Para las empresas pequeñas también observamos un aumento del componente de intensificación, aunque en menor grado que para las empresas grandes. Así, el porcentaje de episodios de I+D de al menos 4 años de duración representa un 38,71 por 100 de los episodios activos y un 46,36 por 100 del valor.

Por último, en las columnas 5 y 6 se presentan el porcentaje y el valor relativo de los episodios de I+D que han durado al menos 10 años. Para las empresas grandes observamos que a pesar de que el porcentaje de episodios que duran al menos 10 años representa el 38,01 por 100 , dichos episodios representan el 52,55 por 100 del valor, lo que indica un importante esfuerzo en el componente de intensificación de la persistencia. Para las empresas pequeñas, en cambio, observamos que solo el 25,81 por 100 de los episodios de I+D alcanzan al menos 10 años de duración y que dichos episodios representan tan solo el 32,12 po 100 del valor de todos los episodios activos. Por tanto, se observa que el esfuerzo de las empresas pequeñas en términos del componente de intensificación es bastante menor que el de las grandes. 
La consideración conjunta de $i$ ) las bajas tasas de persistencia que caracterizan a las empresas pequeñas y ii) el peso relativamente menor en comparación con las empresas grandes que los episodios de larga duración representan sobre el total de gasto en $\mathrm{I}+\mathrm{D}$, sugiere que los episodios de $\mathrm{I}+\mathrm{D}$ de corta duración y aquellos con una probabilidad muy alta de finalización representan una proporción importante del gasto total en I+D en las pequeñas empresas manufactureras.

Si tenemos en cuenta los tres sectores de intensidad tecnológica considerados, se observa que tanto para las empresas pequeñas como para las grandes, el componente de intensificación está positivamente correlacionado con la intensidad tecnológica del sector, siendo especialmente relevante el componente de intensificación de las empresas en sectores de tecnología alta. Además, el proceso de intensificación es siempre mayor para las empresas grandes que para las empresas pequeñas, en todos los sectores de intensidad tecnológica a partir de episodios que duran 4 o más años (excepto para las empresas grandes de tecnología media).

\section{Descomposición de la tasa de crecimiento del gasto en I+D}

En esta sección se describe, en primer lugar, la metodología empleada para calcular la importancia relativa de los márgenes intensivo y extensivo en la evolución del gasto privado en I+D. Posteriormente se analizan los resultados de la aplicación de esta metodología a los datos descritos en las secciones previas, distinguiendo entre empresas grandes-pequeñas y por sector tecnológico. Finalmente, presentamos los resultados de algunos ejercicios de simulación de situaciones hipotéticas con el objetivo de responder a la cuestión de cuánto debería variar la persistencia en la realización de actividades de I+D para poder alcanzar una determinada tasa de crecimiento promedio del gasto privado en I+D.

\subsection{Metodología}

Para un año $t$, podemos expresar el gasto total en I+D como $V_{z j, t}=n_{z j, t} v_{z j, t}$, donde $V_{z j, t}$ es el valor del gasto total en I+D para el año $t$ del sector de intensidad tecnológica $z \in Z$ (donde $Z$ es alta, media y baja) del grupo de tamaño $j \in J$ (donde $J$ es pequeña o grande), $n_{z j, t}$ es el número de episodios de I+D y $v_{z j, t}$ es el valor medio del gasto en I+D para los episodios activos en $t$. En particular, los episodios de I+D pueden clasificarse en dos grupos: los activos tanto en $t-1$ como en $t\left(s_{z, j, t}\right)$ y los nuevos $\left(e_{z j, t}\right)$. Por tanto, el número total de episodios de I+D en $t$ será: $n_{z j, t}=s_{z j, t}+e_{z j, t}$.

De este modo, el aumento del gasto total en I+D para el sector de intensidad tecnológica $z$ del grupo de tamaño $j$ de $t$ a $t+1$ se puede escribir:

$$
\begin{aligned}
& V_{z j, t+1}-V_{z j, t}=n_{z j, t+1} v_{z j, t+1}-n_{z j, t} v_{z j, t}=\left(s_{z j, t+1}+e_{z j, t+1}\right) v_{z j, t+1}-\left(s_{z j, t}+e_{z j, j}\right) v_{z j, t}= \\
& =s_{z j, t+1} v_{z j, t+1}+e_{z j, t+1} v_{z j, t+1}-s_{z j, t+1} v_{z j, t}-d_{z j, t} v_{t}=s_{z j, t+1}\left(v_{z j, t+1}-v_{z j, t}\right)-d_{z j, t} v_{z j, t}+e_{z j, t+1} v_{z j, t+1}
\end{aligned}
$$


donde $s_{z j, t+1}$ es el número de episodios activos tanto en $t$ como en $t+1,\left(v_{z j, t+1}-v_{z j, t}\right)$ es el crecimiento medio del gasto en $\mathrm{I}+\mathrm{D}$ entre estos dos años, $d_{z, j, t}$ es el número de episodios que finalizan en $t, d_{z j, t} v_{z j, t}$ es el valor total del gasto en I+D de los episodios que finalizan en $t, \mathrm{y} e_{z j, t+1} \mathrm{y} v_{z j, t+1}$ son el número de nuevos episodios de $\mathrm{I}+\mathrm{D}$ y su valor total.

Nuestra descomposición se puede refinar si consideramos que la probabilidad de finalización de un episodio de $\mathrm{I}+\mathrm{D}$ en el período $t$ depende de la antigüedad de dicho episodio. Incorporar la antigüedad en la realización de actividades de I+D en la descomposición propuesta en [1], supone estimar las funciones de supervivencia y de riesgo para cada uno de los grupos de tamaño por intensidad tecnológica. Así, podemos definir $s_{z j, t} \equiv\left\{s_{z j, t}^{0}, s_{z j, t}^{1}, s_{z j, t}^{2}, \ldots, s_{z j, t}^{i}, \ldots, s_{z j, t}^{l}\right\}, d_{z j, t} \equiv\left\{d_{z j, t}^{0}, d_{z j, t}^{1}, d_{z j, t}^{2}, \ldots, d_{z j, t}^{i}, \ldots, d_{z j, t}^{l}\right\}$, $v_{z j, t} \equiv\left\{v_{z j, t}^{0}, v_{z j, t}^{1}, v_{z j, t}^{2}, \ldots, v_{z j, t}^{i}, \ldots, v_{z j, t}^{l}\right\}$ y $h_{z j, t} \equiv\left\{h_{z j, t}^{0}, h_{z j, t}^{1}, h_{z j, t}^{2}, \ldots, h_{z j, t}^{i}, \ldots, h_{z j, t}^{l}\right\}$, donde el superíndice $i$ denota la antigüedad del episodio de I+D; $s_{z j, t}^{i}$ es el número de episodios de I+D activos en $t-1$ y en $t$, que no finalizan a lo largo del $i$-ésimo año de realización de forma interrumpida de actividades de I+D; y $h_{z j, t}$ denota la tasa de riesgo correspondiente a un episodio de una empresa del sector de intensidad tecnológica $z$ del grupo de tamaño $j$.

De esta manera podemos reescribir la ecuación [1] del siguiente modo:

$$
V_{z j, t+1}-V_{z j, t}=\sum_{i=1}^{l}[\underbrace{(\underbrace{\left.\left(1-h_{z j, t+1}^{i}\right) n_{z j, t}^{i}\right]}_{\text {Episodios que continúan }} \underbrace{\left[v_{z j, t+1}^{i}-v_{z j, t}^{i}\right]}_{\text {Intensificación }}-\sum_{i=1}^{l}[\underbrace{\left[\left(h_{z j, t+1}^{i} n_{z j, t}^{i}\right) v_{z j, t}^{i}\right.}_{\text {Salidas }}]}_{\text {Margen intensivo }}+\underbrace{\underbrace{e_{z j, t+1} v_{z j, t+1}^{0}}_{\text {Entrantes I+D }}}_{\text {Margen extensivo }}
$$

donde $l$ denota la máxima duración posible de un episodio; $\left(1-h_{z j, t+1}^{i}\right)$ es el porcentaje de episodios que han sobrevivido de $t$ a $t+1 \mathrm{y}\left(1-h_{z j, t+1}^{i}\right) n_{z j, t}^{i}$ nos indica el número de episodios de $\mathrm{I}+\mathrm{D}$ que han sobrevivido de $t$ a $t+1$ (que corresponden a empresas que han realizado ininterrumpidamente actividades de $\mathrm{I}+\mathrm{D}$ por un período de $i$ años); la expresión $\left[v_{z, t+1}^{i}-v_{z j, t}^{i}\right]$ representa el crecimiento (intensificación) del gasto en I+D de las empresas con episodios que siguen activos; $\left(h_{z j, t+1}^{i} n_{z j, t}^{i}\right)$ nos indica el número de episodios de I+D que finalizan en el año $t+1 ;\left(h_{z j, t+1}^{i} n_{z j, t}^{i}\right) v_{z j, t}^{i}$ nos índica su valor total en términos de gasto en $\mathrm{I}+\mathrm{D} ; \mathrm{y} e_{z j, t+1} v_{z j, t}^{0}$ nos da el valor del gasto en $\mathrm{I}+\mathrm{D}$ que corresponde a las empresas que comienzan un nuevo episodio de I+D en el año $t+1$.

La ecuación [2] permite observar que el margen intensivo depende de tres componentes: por una parte, la supervivencia de los episodios de I+D y la intensificación en el gasto de I+D; y, por otra, las empresas que abandonan las actividades de I+D. Así, mayores tasas de supervivencia tienen como resultado más episodios de I+D (un mayor número de episodios continúan activos y menos episodios que finalizan). No obstante, es importante resaltar que la consideración de la antigüiedad de los episodios de I+D es fundamental para nuestro análisis, ya que esperamos que la probabilidad de finalización del episodio sea mayor los primeros años realizando actividades de I+D (dependencia negativa de la duración). Obsérvese también que el último término de 
la ecuación [2] proporciona el margen extensivo y que la suma de la ecuación [2] para los tres sectores tecnológicos considerados nos permite calcular la variación del gasto total privado en $\mathrm{I}+\mathrm{D}$ para cada grupo de tamaño $j$.

\subsection{Resultados empíricos}

En el Cuadro 5 se presenta la contribución porcentual del margen intensivo y extensivo al crecimiento de la I+D corporativa, utilizando la fórmula [2] del apartado anterior. Asimismo, dividimos el margen intensivo en sus dos componentes: la contribución de las empresas que persisten haciendo I+D (persistencia por nivel de intensificación) y la contribución de las empresas que abandonan las actividades de $\mathrm{I}+\mathrm{D}$.

Para las empresas pequeñas, que son las grandes perjudicadas por la crisis, encontramos que el crecimiento negativo de la I+D vendría explicado por el margen intensivo (-360,7 por 100), mientras que el margen extensivo compensaría parte de este deterioro. Además, si analizamos en detalle el margen intensivo encontramos que aunque la contribución de las empresas pequeñas que persisten haciendo I+D es importante y positivo (167,3 por 100), el impacto negativo de aquellas que abandonan $(-528,28$ por 100$)$, más que compensa esta contribución, provocando una contribución final negativa.

El análisis por sector tecnológico revela un patrón similar entre empresas pequeñas de tecnología baja y media: el margen intensivo explicaría el deterioro en el gasto en I+D durante el período de crisis, siendo de especial relevancia el alto impacto negativo de aquellas empresas que abandonan sus actividades de I+D. Las empresas pequeñas de tecnología alta son las únicas capaces de hacer crecer, aunque modestamente, su gasto en I+D. Esto se explica porque la contribución positiva del margen extensivo consigue compensar la contribución negativa del margen intensivo.

En cuanto a las empresas grandes, el principal motor del modesto crecimiento de la I+D durante este período es el margen intensivo (74,4 por 100), mientras que el margen extensivo contribuye en un 25,6 por 100 . Observando los componentes del margen intensivo de las empresas grandes encontramos una contribución positiva de aquellas empresas que persisten (201,4 por 100), que se reduce en un 127 por 100 por aquellas empresas que abandonan las actividades de I+D. No obstante, el análisis por sector tecnológico muestra un comportamiento diferente de los márgenes entre, por un lado, sectores de tecnología baja y media y, por otro, sectores de tecnología alta. Así, para las empresas grandes que pertenecen al primer grupo el margen intensivo es el principal culpable del deterioro en el gasto de $\mathrm{I}+\mathrm{D}$, siendo de nuevo los abandonos el principal determinante de esta caída. Esto significa que las empresas grandes de tecnología alta son las que impulsan el crecimiento global en las empresas de mayor tamaño. Este crecimiento se explica en este caso casi en su totalidad (98 por 100) por el margen intensivo y, dentro de sus componentes, por la persistencia e intensificación de las actividades de I+D. 


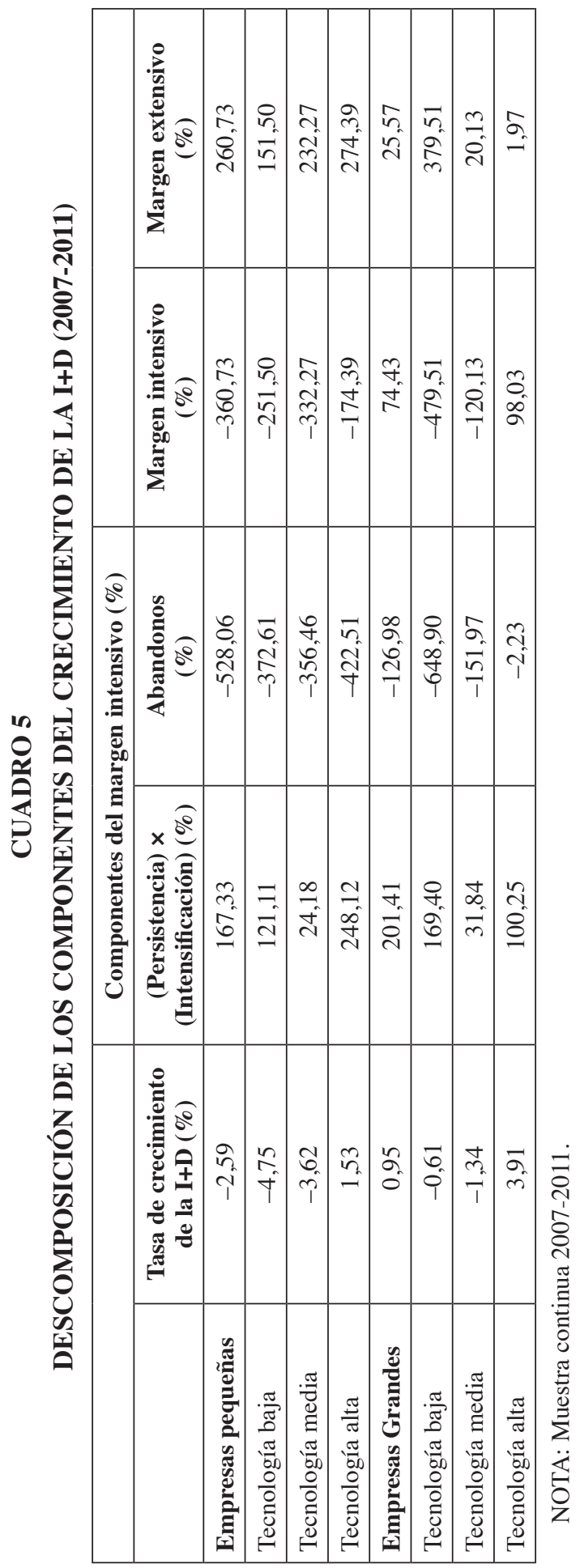


Resumiendo, el comportamiento de la I+D de las empresas pequeñas se caracteriza por altas tasas de rotación (incluso en sectores de tecnología alta) y baja persistencia. El crecimiento negativo del gasto en I+D en el período de crisis viene explicado principalmente por el gran abandono de las actividades de I+D. Solo en el caso de empresas pequeñas de alta tecnología la pérdida de aquellas que abandonan puede compensarse con el aumento de la I+D realizado por las empresas que persisten en sus actividades innovadoras y el gasto realizado por empresas que se inician en tales actividades. Por el contrario, el comportamiento del gasto en I+D de las empresas grandes en el sector manufacturero español se caracteriza por su alta persistencia, con la salvedad de empresas pertenecientes a sectores de tecnología baja. En este período de crisis las grandes impulsoras del crecimiento de la $\mathrm{I}+\mathrm{D}$ en las empresas españolas de mayor tamaño han sido las pertenecientes al sector de tecnología alta, las cuales, a pesar de la baja rotación, han seguido intensificando sus gastos en I+D.

\subsection{Simulaciones}

Tal y como se ha puesto de manifiesto en la sección anterior, la falta de persistencia en la realización de actividades de I+D es el principal factor detrás de la caída en la realización de dichas actividades. Para evaluar la importancia relativa de dicho componente en el crecimiento del gasto en I+D, a continuación llevamos a cabo una serie de ejercicios de simulación orientados a responder a la siguiente pregunta: ¿Cuánto debería variar la persistencia en la realización de actividades de I+D para poder alcanzar una determinada tasa de crecimiento promedio del gasto privado en $\mathrm{I}+\mathrm{D}$ ?

Estas simulaciones nos permiten evaluar la importancia relativa de la persistencia, no solo para cada grupo de empresas en cuanto a tamaño e intensidad tecnológica, sino también entre grupos de empresas. En particular, simularemos cuanto tendría que haber aumentado la persistencia en la realización de actividades de $\mathrm{I}+\mathrm{D}$ para que la tasa de crecimiento de la $\mathrm{I}+\mathrm{D}$ no hubiera sido negativa (la fijaremos en un 0 por 100) en aquellos casos en los que obtenemos tasas negativas. Obsérvese que el ejercicio de simulación no contempla los casos en que dicha tasa fue positiva ${ }^{13}$. Estos resultados se presentan en el Cuadro 6.

En primer lugar analizamos el caso de las empresas pequeñas, sin distinguir por sectores tecnológicos. Estas empresas han experimentado una caída en la tasa de crecimiento (media anual) de la I+D del 0,026 por 100 durante el período analizado. Para este grupo de empresas, el componente de persistencia en la realización de actividades de I+D tendría que haber sido del 16,96 por 100 para que la tasa de crecimiento de la $\mathrm{I}+\mathrm{D}$ no hubiera sido negativa (en un $-0,026$ por 100 ). En otras

\footnotetext{
${ }^{13}$ Las empresas grandes, en general, y el sector de tecnología alta, tanto en el grupo de empresas grandes como en el de pequeñas, presentan tasas positivas a lo largo del período 2007-2011. Estos son los casos para los que no hemos realizado el ejercicio de simulación.
} 


\section{CUADRO 6}

\section{EJERCICIOS DE SIMULACIÓN NUMÉRICA DEL COMPONENTE DE PERSISTENCIA EN EL CRECIMIENTO DE LA I+D}

\begin{tabular}{|c|c|c|c|}
\hline & $\begin{array}{c}\text { Crecimiento de la } \\
\text { I+D }(\%)\end{array}$ & $\begin{array}{c}\text { Tasa de crecimiento } \\
\text { de la } I+D=0 \%\end{array}$ & Persistencia (\%) \\
\hline \multicolumn{4}{|l|}{ Total } \\
\hline Empresas pequeñas & $-0,026$ & 0 & 16,962 \\
\hline Empresas grandes & 0,947 & - & - \\
\hline \multicolumn{4}{|l|}{ Empresas pequeñas } \\
\hline Tecnología baja & $-4,754$ & 0 & 22,135 \\
\hline Tecnología media & $-3,616$ & 0 & 20,827 \\
\hline Tecnología alta & 1,534 & - & - \\
\hline \multicolumn{4}{|l|}{ Empresas grandes } \\
\hline Tecnología baja & $-0,608$ & 0 & 15,406 \\
\hline Tecnología media & $-1,336$ & 0 & 49,900 \\
\hline Tecnología alta & 3,914 & - & - \\
\hline
\end{tabular}

palabras, para que la tasa promedio de crecimiento del gasto en I+D durante la crisis hubiera sido del 0 por 100, el componente de persistencia en la realización de I+D por aquellas empresas que innovan continuamente tendría que haber aumentado en un 16,96 por 100 . Este resultado indica que la persistencia es el componente más débil entre las empresas pequeñas y confirma la evidencia obtenida en el análisis descriptivo, en el que observábamos que la persistencia en la realización de actividades de I+D era mucho más baja para las empresas pequeñas que para las grandes. Desde otro punto de vista, este resultado indica que para lograr un aumento significativo en el crecimiento de la I+D de las empresas pequeñas sería necesario que realizaran un considerable esfuerzo en la persistencia en la realización de actividades de I+D.

En todo caso, estos resultados no son uniformes por sectores tecnológicos. Hemos analizado únicamente los sectores de tecnología baja y media, tanto para las empresas grandes como pequeñas, puesto que son los que experimentan crecimiento negativo en la realización en actividades de I+D. Así, para las empresas pequeñas, el componente de persistencia debería haber aumentado un 22,14 por 100 y un 20,83 por 100 , para los sectores de tecnología baja y media, respectivamente, para que la tasa de crecimiento de la I+D hubiera sido del 0 por 100. En cambio, para las empresas grandes este componente debería haber aumentado un 15,41 por 100 y un 49,9 por 100 para los sectores de tecnología baja y media, respectivamente. 


\section{Conclusiones}

El objetivo de este artículo ha sido estudiar el crecimiento del gasto corporativo en I+D en el sector manufacturero español a partir del análisis del comportamiento del margen extensivo e intenso durante el período de crisis. Para ello utilizamos datos de la Encuesta sobre Estrategias Empresariales sobre los gastos de I+D de las empresas manufactureras españolas durante el período 2007-2011, aunque parte del estudio también utiliza datos desde 1990. Nuestros resultados señalan que las empresas difieren en sus actividades de I+D a lo largo de cada margen según su tamaño y el sector tecnológico al que pertenecen. Así, el comportamiento de la I+D de las empresas pequeñas se caracteriza por altas tasas de rotación y persistencia baja. El importante retroceso en el gasto en I+D en las empresas pequeñas se explica principalmente por el fuerte peso de aquellas empresas que cesan su actividad innovadora, que salvo en el caso de empresas pertenecientes al sector de tecnología alta, es incapaz de verse compensado por la I+D realizada por empresas que se inician en este tipo de actividades. Por el contrario, el comportamiento de la I+D de las empresas grandes se caracteriza por su alta persistencia. Las empresas grandes han visto aumentar su gasto en I+D durante el período de crisis, aunque de forma modesta. Este aumento, ha venido impulsado por las empresas de alta tecnología, en las que tanto el margen extensivo, pero sobre todo el margen intensivo, tienen una contribución positiva.

Las actividades de I+D gozan de elevada importancia en la agenda política de los gobiernos a nivel nacional, europeo y regional. Los gastos de I+D en España han sido claramente inferiores a la media de la OCDE y Europa, y han retrocedido significativamente en el período de crisis; por lo tanto, alejándonos todavía más del objetivo de la Agenda de Lisboa (alcanzar un gasto en I+D del 3 por 100 del PIB). Los resultados de los ejercicios de simulación permiten esbozar algunas recetas de política económica con el fin de impulsar estas actividades. En primer lugar, se necesitan iniciativas de política en innovación diseñadas según el tamaño y el sector tecnológico de la empresa. En segundo lugar, nuestros resultados indican que las políticas destinadas a mejorar la tasa de intensificación parecen ser más eficaces, ya que solo es necesario un modesto incremento en la tasa de intensificación para alcanzar el objetivo deseado. Sin embargo, esto solo tendrá efectos a corto plazo para las empresas pequeñas, ya que a menos que estas tengan el incentivo adecuado para continuar invirtiendo en $\mathrm{I}+\mathrm{D}$, cualquier ganancia en el componente de intensificación o iniciación desaparecerá en pocos años. Sin duda, la crisis ha demostrado la vulnerabilidad de las empresas pequeñas a continuar realizando sus actividades de I+D. Por ello, una política anticíclica de innovación dirigida tanto a evitar el alto abandono de las empresas pequeñas como a intensificar su inversión en I+D nos hubiese permitido seguir alcanzando los objetivos de convergencia en materia de I+D. 


\section{Referencias bibliográficas}

[1] AÑÓN HIGÓN, D., MÁÑEZ CASTILLEJO, J.A. y SANCHIS LLOPIS, J.A. (2011). «Iniciación, persistencia e intensificación en las actividades de I+D en España». Economía industrial, 382, 13-24.

[2] BERNARD, A.B., JENSEN, B.J., REDDING, S.J. y SCHOTT, P.K. (2009). «The Margins of U.S. Trade». American Economic Review, 99(2), 487-93.

[3] BESEDES, T y PRUSA, T.J. (2007). «The Role of Extensive and Intensive Margins and Export Growth». NBER Working Papers 13628, National Bureau of Economic Research, Inc.

[4] BLUNDELL, R., GRIFFITH, R. y VAN REENEN, J. (1995). «Dynamic Count Data Models of Technological Innovation», The Economic Journal, 105(429), 333-344.

[5] CEFIS, E. (2003). «Is there persistence in innovative activities?». International Journal of Industrial Organization, 21(4), 489-515.

[6] COMÍN, D., HOBIJN, B. y ROVITO, E. (2008). «A new approach to measuring technology with an application to the shape of the diffusion curves». Journal of Technology Transfer, 33, 187-207.

[7] COTEC (2014). Informe Cotec 2014: Tecnología e Innovación en España. Fundación Cotec.

[8] CUERVO-CAZURRA, A. y UN, C.A. (2010). «Why some firms never invest in formal R\&D». Strategic Management Journal, 31(7), 759-779

[9] DUGUET, E. y MONJON, S. (2004). «Is innovation persistent at the firm level? An econometric examination comparing the propensity score and regression methods». Cahiers de la Maison des Sciences Economiques v04075, Paris

[10] FARIÑAS, J.C. y JAUMANDREU, J. (1999). «Diez años de Encuesta sobre Estrategias Empresariales». Economía Industrial, 329, 29-42.

[11] GEROSKI, P. A., VAN REENEN, J. y WALTERS, C. F. (1997). «How persistently do firms innovate?». Research Policy, 26(1), 33-48.

[12] HELPMAN, E., MELITZ, M. y RUBINSTEIN, Y. (2008). «Estimating trade flows: Trading partners and trading volumes». Quarterly Journal of Economics, 123, 441487.

[13] HUMMELS, D. y KLENOW, P. (2005). «The Variety and Quality of a Nation's Exports» . American Economic Review, 95, 704-723.

[14] MANSFIELD, E. (1968). Industrial Research and Technological Innovation: An Econometric Analysis, New York.

[15] MÁÑEZ, J.A., ROCHINA-BARRACHINA, M., SANCHIS-LLOPIS, A. y SANCHISLLOPIS, J.A. (2009). «The role of sunk cost in the decision to invest in R\&D». The Journal of Industrial Economics, 57(4), 712-735.

[16] MÁÑEZ, J.A., ROCHINA-BARRACHINA, M., SANCHIS-LLOPIS, A. y SANCHISLLOPIS, J.A. (2015). «The determinants of R\&D persistence in SMEs». Small Business Economics, 44, 505-528.

[17] OCDE (2003). Science, Technology and Industry Scoreboard. OCDE, Paris.

[18] OCDE (2014). Main Science and Technology Indicators. Vol 2014/1. OCDE, Paris.

[19] PETERS, B. (2009). «Persistence in innovation: stylised facts and panel data evidence», Journal of Technological Transfer, 34(2), 226-243. 
[20] RAYMOND, W., MOHNEN, P., PALM, F. y SCHIM VAN DER LOEFF, S. (2010). «Persistence of innovation in Dutch manufacturing: Is it spurious?». The Review of Economics and Statistics, 92(3), 495-504.

[21] ROGERS, M. (2004). «Networks, firm size and innovation». Small Business Economics, 22(2), 141-153.

[22] ROSENBERG, N. (1976). Perspectives on Technology. Cambridge: Cambridge University Press.

[23] SUTTON, J. (1991). Sunk Costs and Market Structure. Cambridge, Massachusetts: The MITT Press. 